\title{
Uncommon Case of Ureteral Clip Migration Following Laproscopic Hysterctomy
}

\author{
Marwane Andaloussi Benatiya ${ }^{\mathrm{a}}$, Azeddine El Kiassi ${ }^{\mathrm{a}}$, \\ Ghizlane Rais ${ }^{\mathrm{b}, \mathrm{c}}$
}

\begin{abstract}
Inadvertent ureteric injury is a hazard of laparoscopic hysterectomy and may cause usually ureteric fistulae or obstruction. Ureteral clip migration following laparoscopic hysterectomy as iatrogen ureteric injury has never been described. Here we report an unusual case of a 45-year-old female who presented with right flank pain. She has an history of laparoscopic hysterectomy 1 year ago. Abdominopelvic computed tomography (CT) scan confirmed the diagnosis of right renal colic following migration of a metalic clip. The patient was treated by the ureteroscopic ablation of the clip with the use of double $\mathrm{J}$ stent and had successful outcomes.
\end{abstract}

Keywords: Surgical clip migration; Ureter; Laparoscopic hysterectomy

\section{Introduction}

The majority of ureteral injuries are iatrogenic in nature. Such injuries are often known to occur most frequently during urology, gynecology and general surgery $[1,2]$. However, ureteral obstruction due to surgical clip migration into the ureter is an extremelly rare phenomenon $[1,2]$. We report here an interesting case of spontaneous migration of metal clip into the ureter in a female patient that underwent laparoscopic hysterectomy 1 year previously for polymyomatous uterus. To the best of our knowledge, this is the first case decribed in a patient after laparoscopic hysterectomy. The purpose of presenting this case report is not only to report an uncommon case of surgical clip migration, but also to raise awareness among clinicians that this rare entity can occurs after laparoscopic hysterectomy.

Manuscript submitted June 15, 2018, accepted June 27, 2018

aDepartment of Urology, Hassan 2 Hospital, Agadir, Morocco

${ }^{b}$ Medical Oncology Department, Regional Center of Oncology, Agadir, Morocco

${ }^{\mathrm{c} C}$ Corresponding Author: Ghizlane Rais, Medical Oncology Department, Regional Center of Oncology, Agadir, Morocco. Email: medghiz@hotmail.com

doi: https://doi.org/10.14740/jmc3114w

\section{Case Report}

A 45-year-old lady presented with repeated episodes of right flank pain since 3 weeks. Her past history was significant for laparoscopic hysterectomy for polymyomatous uterus 1 year ago. Plain radiography of the abdomen revealed a $5-\mathrm{mm}$ opacity projected over the pelvic portion of the right ureter (Fig. 1). The renal ultrasonography showed moderate right ureterohydranephrosis without detectable lithiasis. Abdominopelvic computed tomography scan revealed a 0.5 -cm opacity (arrow) on the right lower ureter (Fig. 2).

Ureteroscopy with the patient under general anesthesia showed metalic foreign body at the lower right ureter (Fig. 3) with incomplete stenosis that was dilated by a balloon. The medium-sized surgical clip was identified and removed using a dormia basket device and a double $\mathrm{J}$ stent was placed for 2 months. At 3-month follow-up, the patient was asymptomatic with absolutely normal renal function test and absence of ureterohydonephrosis in abdomen ultrasonography.

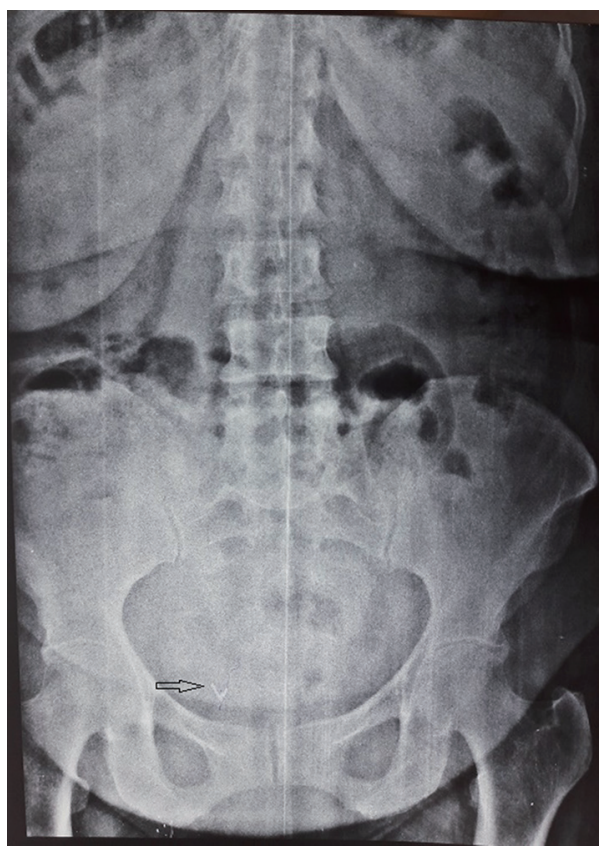

Figure 1. Plain X-ray of the abdomen revealed metallic surgical clips (arrow) on the right lower abdomen. 


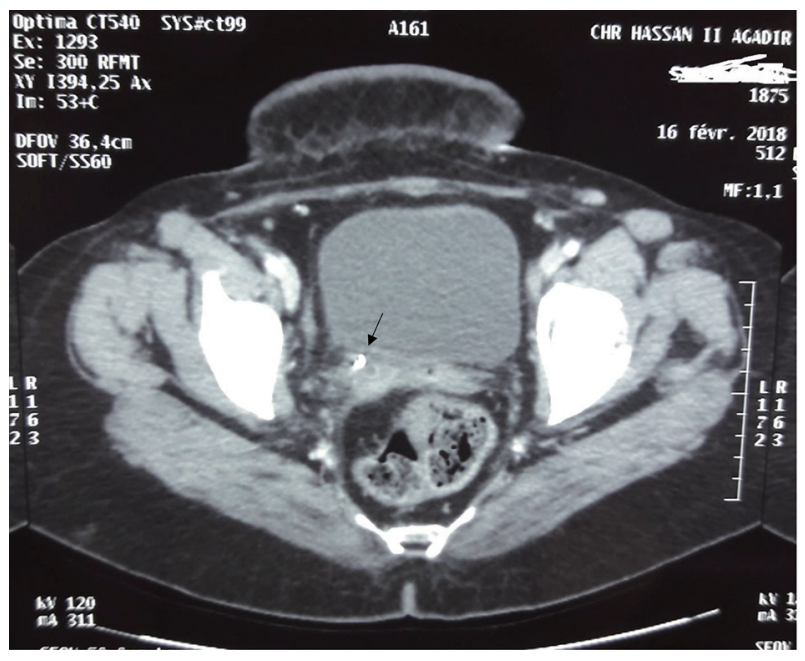

Figure 2. Abdominopelvic computed tomography scan revealed a 0.5$\mathrm{cm}$ opacity (arrow) on the right proximal ureter.

\section{Discussion}

Surgical clips have been used for many years in a variety of surgical procedures and they have proven competency and efficacy. The number of the surgical clips used is variable and unpredictable which depends on the procedure. However, regardless of their type, migration of the surgical clips away from the surgical site is a well-known phenomenon that occurs quite regularly.

While migration of clips into the common bile duct has been previously reported in several laparoscopic cholecystectomies [3, 4], migrations of surgical materials into the urinary tract like pelvicalyceal system, ureter and bladder are a rare condition. It has been reported following various urological procedures either in the ureter after laparoscopic partial nephrectomy [5-7] or into the bladder after surgery laparoscopic radical prostatectomy $[5,8]$. Moreover, a case of intrarectal migration after prostatectomy [7] and a case of migration in the colon after partial nephrectomy [9] have also been reported.

This case is very interesting because to the best of our knowledge, this is the first described case of migration of surgical clip into the ureter after laparoscopic hysterectomy.

It was suggested that migrated clips are more often associated with laparoscopic procedures $[5,6]$. This implies that we would expect to see more of migrated clips with the vast preferred use of laparoscopy

The exact sequence of events leading to clip migration is poorly understood, but is thought to be partly affected by technical factors such as correct placement and use of minimal numbers of clips [5]. A proposed mechanism of clip migration after cholecystectomy involved the compression of the clipped cystic duct by the liver. The cystic duct and clips then become inverted into the lumen of the common bile duct. Over time, this structure becomes necrotic, and the clips fall away into the common bile duct. The number of clips used during the initial surgery is also an important factor [3,4]. In our patient, the non-absorbable surgical clip probably eroded into the ureter which has obstructed subsequently probably due to chronic inflammatory reactions, and caused ureterohydronephrosis.

The median time of clip migration is usually 2 years, but it can vary from 11 days to 20 years, while the median number of migrated clip is 1 (range: 1 - 6) [5]. In this patient, clip migration was diagnosed 1 year after laparoscopic hysterectmy which concord with the literature.

The choice of treatment is based on the location, type and extend of ureteral injury caused by migrated clip [10].There is a report of the migration of absorbable Lapra-Ty suture clips in the collecting system after laparoscopic partial nephrectomy [10], and Massoud [11] also reported the migration of a metal surgical clip into the ureter after open partial nephrectomy, all of which were passed spontaneously. The majority of patients with delayed diagnosed ureteral injuries should be managed by an initial endourological approach.

To improve the management of ureteric injury, there must be a high index of suspicion, especially after laparoscopic operations. Most reported cases were treated with endourological treatment which yields acceptable results.

\section{Conclusions}

Surgical clips may migrate postoperatively and cause second-

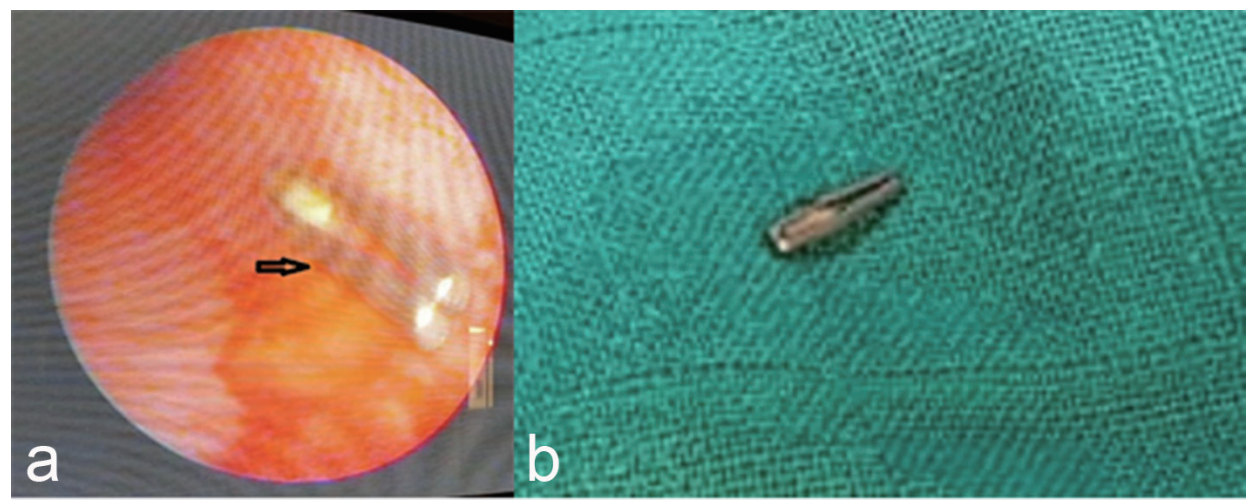

Figure 3. (a) Ureteroscopy showed a metallic parallelepiped foreign body at the lower right ureter. (b) The foreign body was removed by use of a ureteroscopic stone basket device and was identified as a metallic unresorbable clip. 
ary complications. Therefore, the surgeon must be aware of the possibility of clip migration. Furthermore, clips should be absorbable and applied selectively over vessels and far from the collecting system.

\section{Conflict of Interest}

The authors declare that they have no competing interests.

\section{References}

1. Elliott SP, McAninch JW. Ureteral injuries from external violence: the 25-year experience at San Francisco General Hospital. J Urol. 2003;170(4 Pt 1):1213-1216.

2. Selzman AA, Spirnak JP. Iatrogenic ureteral injuries: a 20-year experience in treating 165 injuries. J Urol. 1996;155(3):878-881.

3. Dell'Abate P, Del Rio P, Soliani P, Colla G, Sianesi M. Choledocholithiasis caused by migration of a surgical clip after video laparoscopic cholecystectomy. J Laparoendosc Adv Surg Tech A. 2003;13(3):203-204.

4. Yoshizumi T, Ikeda T, Shimizu T, Ohta S, Nagata S, Sonoda T, Sugimachi K. Clip migration causes choledocho- lithiasis after laparoscopic cholecystectomy. Surg Endosc. 2000;14(12):1188.

5. Wu SD, Rios RR, Meeks JJ, Nadler RB. Rectal Hem-oLok clip migration after robot-assisted laparoscopic radical prostatectomy. Can J Urol. 2009;16(6):4939-4940.

6. Shrivastava P, Nayak B, Singh P. Migrated Hem-o-Lok clips in the ureter: a rare cause of recurrent urinary tract infection. BMJ Case Rep. 2017;2017.

7. Fiard G, Peneau M, Soulimane B. [Massive migration of Hem-o-lok(R) clips following open partial nephrectomy: a case report and literature review]. Prog Urol. 2014;24(10):616-619.

8. Mora ER, Gali OB, Garin JA, Arango O. Intravesical migration and spontaneous expulsion of a Hem-o-lok polymer ligating clip after laparoscopic radical prostatectomy. Urology. 2010;75(6):1317.

9. Vedel PF, Wittendorf HE, Norus TP. Migration of clips to the colon after laparoscopic partial nephrectomy. BMJ Case Rep. 2017;2017.

10. Miller M, Anderson JK, Pearle MS, Cadeddu JA. Resorbable clip migration in the collecting system after laparoscopic partial nephrectomy. Urology. 2006;67(4):845 e847-848.

11. Massoud W. Spontaneous migration of a surgical clip following partial nephrectomy. Urol J. 2011;8(2):153-154. 